\title{
Qualis Periódicos e a produção de capital científico nos Programas de Pós-Graduação em Educação Física
}

\author{
Priscilla Maia da Silva* \\ Jeane Barcelos Soriano**
}

\begin{abstract}
Resumo: Objetivo: verificar nos documentos oficiais da Avaliação Trienal dos Programas de Pós-Graduação (PPGs) em Educação Física (EF) (triênios de 2007 e 2010) o funcionamento do Qualis Periódicos (Qualis P) na determinação de regras objetivas para obtenção de capital científico. Metodologia: a abordagem qualitativa e o estudo do tipo exploratório foram os meios mais adequados para a condução do estudo. Selecionamos seis PPGs em EF. Coletamos os dados nos comentários dos avaliadores em doze Fichas da Avaliação Trienal de 2007 e 2010. Conclusão: o Qualis P funciona como estrutura estruturada estabelecendo o princípio lógico de acúmulo de capital científico.
\end{abstract}

Palavras-chave: Avaliação. Produção Científica. PósGraduação. Educação Física.

\section{INTRODUÇÃo}

No Brasil, a pós-graduação é considerada o lócus da produção de conhecimento científico e formação de pesquisadores. A Coordenação de Aperfeiçoamento de Pessoal de Nível Superior (CAPES), além de realizar a avaliação dos cursos de mestrado e doutorado que compõem os Programas de Pós-Graduação (PPGs), atua em conjunto com o Conselho Nacional de Desenvolvimento

\footnotetext{
"Priscilla Centro de Educação Física e Esporte. Universidade Estadual de Londrina. Londrina, PR, Brasil.E-mail: primaiasilva@gmail.com

"Jeane Centro de Educação Física e Esporte. Universidade Estadual de Londrina. Londrina, PR, Brasil.E-mail: jeane@uel.br
} 
Científico e Tecnológico $(\mathrm{CNPq})$ no fomento à pesquisa e pósgraduação. O conjugado CAPES - CNPq transformam o governo brasileiro no principal promotor e financiador da produção científica e formação de pesquisadores. Assim, a projeção internacional do país no contexto científico internacional é resultado da expansão e consolidação do Sistema Nacional de Pós-Graduação vinculada ao "crescimento da ciência brasileira, expressa sobretudo pelos progressos na produção científica, aferida pela publicação de artigos em periódicos de circulação internacional indexados" (BRASIL, 2010, p.223).

O sistema Qualis Periódicos (Qualis P) exerce um papel fundamental neste processo. O Qualis P é o instrumento definidor de critérios para classificação da produção de artigos, sendo empregado na estratificação da produção científica dos PPGs de todas as áreas de conhecimento. A fonte de informações para composição do Qualis P é coletada a partir da disseminação de artigos dos PPGs. A classificação e consulta da lista hierárquica dos periódicos de cada área de conhecimento é realizada pelo aplicativo Webqualis (COORDENAÇÃO..., 2013b). O artigo produzido pelo PPG é classificado conforme a posição que o periódico ocupa no Webqualis. Assim, cada área de conhecimento tem seu Webqualis com a lista dos periódicos ranqueados conforme o parâmetro de classificação divulgado no Qualis P de cada área (COMISSÃO..., , 2009; TANI, 2007; VITOR-COSTA; MAIA DA SILVA; SORIANO, 2012; MARCHLESWKI; MAIA DA SILVA; SORIANO, 2011).

Como as bases de indexação de periódicos do Institute for Scientific Information (ISI) e Scopus são referências de excelência no cenário científico internacional, o sistema de ranqueamento empregado por estas bases também são utilizados para compor os critérios de classificação do Qualis P. O ISI, da Thomson Reuters, é responsável pela base Web of Science (WoS). A WoS adota o indicador bibliométrico (IB) baseado em citação conhecido como Fator de Impacto (FI) para elaborar o ranking Journal of Citation Reports (JCR). A Elsevier mantém a base Scopus e usa o índice h 
para compor o SCImago Journal Rank (SJR) (BUTLER, 2008; VITOR-COSTA; MAIA DA SILVA; SORIANO, 2012). A Educação Física (EF) adotou o FI desde a implantação do Qualis P na Avaliação Trienal dos PPGs - triênio 1998-2000 (SOUZA; PAULA, 2002). A partir da Avaliação Trienal de 2007-2009, o índice $\mathrm{h}$ também foi incorporado ao Qualis P da EF (COMISSÃO..., 2009). $\mathrm{O}$ FI e o índice $\mathrm{h}$ foram utilizados para classificar periódicos nas duas melhores posições do Qualis P (COMISSÃO..., 2007a; 2009).

As publicações em rankings de instituições prestigiadas no campo científico são a melhor maneira de atribuir crédito simbólico. Com isso, os IB que usam a citação como métrica vêm sendo considerados como indicadores de prestígio científico e, portanto, de capital científico (BOURDIEU, 2004; ROMANCINI, 2006). Os resultados obtidos em instituições prestigiadas mundialmente, como o ISI e Scopus, são bons indicadores de capital científico.

Independente do modo de cálculo de obtenção do FI ou índice $\mathrm{h}$ do periódico, nos dois IB o valor não é necessariamente representativo das citações de todos os artigos. Um artigo pode passar pela revisão de pares e ser publicado em um periódico com alto FI/ índice $\mathrm{h}$, mas isso não garante que será citado (VITOR-COSTA; MAIA DA SILVA; SORIANO, 2012; MAIA DA SILVA; KUBO; CAMATA; SORIANO, 2013 ). Ainda que o artigo não seja citado, tem-se atribuído ao processo de revisão por pares a garantia da qualidade do artigo aprovado. Todavia, o mainstream da produção científica de cada área de conhecimento pode influenciar no processo de tomada de decisão do parecerista (efeito Halo), colocando em dúvida a "suposta" neutralidade do peer review (DAVYT; VELHO; 2000; VITOR-COSTA; MAIA DA SILVA; SORIANO, 2012).

Reconhecendo que o Qualis P é "reorganizado em conformidade com a fluência e lutas do campo científico" (VITOR-COSTA; MAIA DA SILVA; SORIANO, 2012, p.583), o cenário competitivo da produção científica nos levou a adotar o conceito de campo científico, de Pierre Bourdieu. O campo científico, como um campo social, envolve interesses que vão muito além da contribuição do conhecimento produzido para o avanço de uma área de 
conhecimento. Para Bourdieu, o que está em jogo no campo científico, além da busca por prestígio social obtida com o reconhecimento dos pares-concorrentes, é a disputa por posições sociais que garantem a autoridade de definir o que é (e o que não é) científico. Em outras palavras, o campo científico é um espaço no qual se tem uma luta por posições dominantes. A posição dominante é obtida com a posse de alto índice de capital científico e do conhecimento sobre o funcionamento das regras objetivas do campo o que, por sua vez, garante o poder de estabelecer os limites do campo dos problemas, dos métodos e das teorias que os dominantes no campo consideram científicas (BOURDIEU, 1975).

A ordem científica só pode ser estabelecida com um conjunto de instituições que garantem a (re)produção e circulação dos bens científicos. Os periódicos prestigiados cumprem seu papel como instituição reprodutora da lógica científica dominante. Os artigos consagrados são aqueles aprovados com critérios de seleção emparelhados com os princípios da ciência considerada oficial. Para manter a ordem científica, produções que não estão em sintonia com a ciência oficial são censuradas ou desencorajadas. Também, o sistema de formação de futuros pesquisadores garante a preparação de novatos dentro da ordem científica dominante no campo científico (BOURDIEU, 1975).

Como no Brasil os PPGs são responsáveis pela produção científica e formação de pesquisadores, podemos supor que o campo científico se desenvolve na pós-graduação. Supostamente, o conjugado CAPES-CNPq, como instituições, garantem a conservação da lógica científica dominante no campo científico brasileiro, impactando nas áreas de conhecimento (campos científicos). Por sua vez, o corpo docente e discente está sujeito a lógica científica que rege a produção de conhecimento nos PPGs. Com a pressão que os PPGs sofrem para disseminar o conhecimento produzido em periódicos com FI/índice h, "não só docentes, mas os discentes também acabam por focarem na disseminação no formato de artigos" (VITOR-COSTA; MAIA DA SILVA; SORIANO, 2012, p.589). 
Adotamos como hipótese que o Qualis P, como um instrumento de classificação da produção intelectual dos PPGs e, parte da avaliação dos PPGs em EF, representa o estado objetivado da regra de acúmulo de capital científico vigente no campo científico da EF. O objetivo foi verificar nos documentos oficiais da Avaliação Trienal dos PPGs em EF (triênios de 2007 e 2010) o funcionamento do Qualis Periódicos na determinação de regras objetivas para obtenção de capital científico.

\section{Decisões metodológicas}

A abordagem qualitativa e a pesquisa exploratória foram os meios mais adequados para condução do estudo (MINAYO, 1998; RICHARDSON, 2008; CRESWELL, 2007; DENZIN; LINCON, 2006). Os dados foram coletados nas justificativas realizadas pela Comissão de Avaliação da Área 21 - Educação Física (CA-EF) nas Fichas de Avaliação (FA) de dois triênios: período 2004-2006 Avaliação Trienal de 2007 (AT-2007); e período 2007-2009 - Avaliação Trienal de 2010 (AT-2010) (COMISSÃO DE AVALIAÇÃO EDUCAÇÃO FÍSICA, 2007b; 2007c; 2007d; 2007e; 2007f; 2007g; 2010b; 2010c; 2010d; 2010e; 2010f; 2010g). Analisamos os resultados da AT-2007 e AT-2010, pois a Classificação de Livros foi incorporada a partir da AT-2007, o que permitiu verificar, entre os dois instrumentos de classificação da produção intelectual, a força do Qualis P na determinação das regras objetivas de obtenção de capital científico.

Utilizamos como documento de apoio o instrumento definidor do critério de classificação da produção de artigos dos PPGs da EF (Qualis P). O Qualis P da EF foi organizado na AT-2007 em: IA, IB; IC (Internacional); NA; NB e NC (Nacional). A posição IA e IB foram as posições mais altas, com FI. Na AT-2010 o Qualis P sofreu alteração no formato e ficou classificado por estratos: A1 e A2 (mais altos, com FI ou índice h), B1; B2; B3; B4; B5 e C (COMISSÃO..., 2007a; 2009). Também utilizamos o Documento e Relatório de Área da AT-2007 e AT-2010, elaborado pela CA-EF com os principais pontos resultantes da Avaliação Trienal (COMISSÃO..., 2007a; 2009; 
2010a). Por fim, o Caderno de Indicadores - Proposta do Programa, preenchido pelo PPG e encaminhado para a CAPES (COORDENAÇÃO..., 2013a).

A seleção dos PPGs da EF foi realizada com base na sistemática de avaliação empregada pela CAPES, que tem na internacionalização o principal elemento de atribuição de qualidade. A CAPES realiza a avaliação a cada três anos, atribuindo notas de 1 a 7. A nota mínima para abertura de um PPG é 3 e as notas 6 e 7 são específicas para PPGs com parcerias internacionais que resultam em publicações de artigos em periódicos do exterior, representando seu padrão de excelência internacional. O resultado é o ranking oficial dos PPGs, por área de conhecimento (CENTRO..., 2010; COORDENAÇÃO..., , 2006; BRASIL, 2010). Para a seleção dos PPGs, utilizamos o seguinte critério: obter nota alta na AT-2007 e/ou AT-2010 (COMISSÃO..., 2007b; 2007c; 2007d; 2007e; 2007f; 2007g; 2010b; 2010c; 2010d; 2010e; 2010f; 2010g). A nota alta indica excelência dos PPGs na produção científica e formação de pesquisadores, pois contam com cursos de doutorado (BRASIL, 2010). Também, demonstra como os PPGs que ocupam o topo do ranking na avaliação da CAPES se ajustam conforme as regras de produção do capital científico.

Nenhum PPG da EF obteve nota 7 na AT-2007 e AT-2010. Assim, selecionamos seis PPGs com nota 5 ou 6 e analisamos 12 FA (6 FA de cada triênio). A seguir, consta o nome do PPG, universidade, acrônimo e nota na AT-2007 e AT-2010 dos PPGs selecionados (COMISSÃO..., 2007b; 2007c; 2007d; 2007e; 2007f; 2007g; 2010b; 2010c; 2010d; 2010e; 2010f; 2010g):

Educação Física - Universidade de São Paulo - PPGEF/USP $(6 / 6)$;

Ciências da Motricidade - Universidade Estadual Paulista Júlio de Mesquita Filho - PPGCM/UNESP (5/6);

Educação Física - Universidade Federal de Santa Catarina PPGEF/UFSC (5/5); 
Ciências do Movimento Humano - Universidade Federal do Rio Grande do Sul - PPGCMH/UFRGS (4/5);

Educação Física - Universidade Federal do Paraná - PPGEF/ UFPR (4/5);

Educação Física - Universidade Gama Filho - PPGEF/UGF $(5 / 4)$.

Lawrence Bardin (1977) foi a referência que norteou os passos empregados na análise. Realizamos o recorte de conteúdo nos comentários dos avaliadores presentes nas FA. Organizamos os recortes em três unidades de contexto: (1) recortes da produção científica do corpo docente (RCDo); (2) recortes da produção científica do corpo discente (RCDi); e (3) participação conjunta do corpo docente e discente na produção científica (RCDoDi). Elaboramos uma planilha no Excel para identificar a frequência de aparição (1) ou ausência (0), nos recortes, de seis características relacionadas com a produção científica dos PPGs em EF (características foram descritas durante a discussão dos dados). Para a escolha das características, consideramos a principal mudança na avaliação da produção intelectual dos PPGs da área 21, da AT-2007 para a AT-2010, a saber: "maior importância para a inserção internacional" (COSTA, 2009, p.48). Organizamos os dados em uma categoria temática, definida a posteriori. Com base na Teoria Geral dos Campos Sociais, de Pierre Bourdieu (1975; 1983; 2004; 2007a; 2007b; 2011), o "capital científico" foi definido como núcleo de sentido para a categoria temática discutida neste artigo.

\section{Qualis P e os pontos CaRdeais da PROdução de CONHECIMENTo Dos PPGs em EF}

A Figura 1 apresenta a frequência de quatro características vinculadas ao Qualis P e Classificação de Livros no RCDo e RCDi: 
Figura 1 - Frequência relativa $(\%)$ de quatro características relacionadas à produção científica do corpo docente e discente nas 12FA analisadas (AT-2007 e AT-2010)

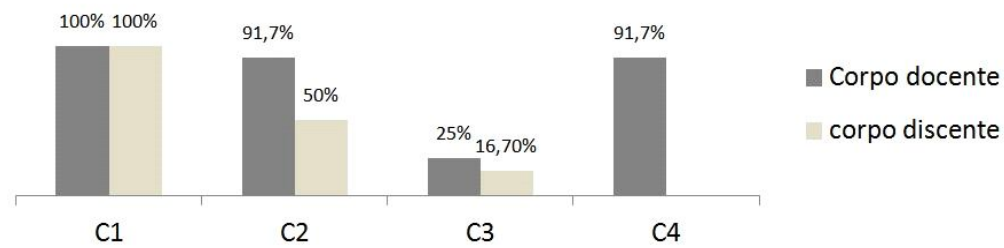

Fonte: Adaptado dos Resultados da Avaliacão Trienal 2007 e 2010. Fichas de Avaliação do PPGEF/USP, PPGCM/UNESP, PPGEF/UFSC, PPGCMH/UFRGS, PPGEF/UFPR e PPGEF/UGF (COMISSÂO..., 2007b; 2007c; 2007d; 2007e; 2007f; 2007g; 2010b; 2010c; 2010d; 2010e; 2010f; 2010g).

Os seguintes termos foram considerados para cada característica:

C1 - Publicação de artigos classificados no Qualis P: IA; IB; IC; NA; NB; NC (AT-2007); A1; A2; B1; B2; B3; B4; B5; C (AT2010); Qualis; estrato; publicações em periódicos; periódicos de boa indexação; publicações de artigo.

C2 - Publicação de artigos em periódicos com FI/índice h: IA; IB (AT-2007); A1; A2 (AT-2010); artigos de impacto internacional; estratos mais elevados; estratos superiores; estratos altos; periódicos indexados internacionalmente; produção internacional.

C3 - Publicação qualificada de livros/capítulos: qualquer destaque realizado pela CA-EF para a qualificação da produção de livros/capítulos. Menção unicamente a contagem de livros e capítulos não foi considerada.

C4 - Bolsista PQ: Bolsa de Produtividade em Pesquisa (Bolsa $\mathrm{PQ}$ ), atribuída pelo CNPq aos pesquisadores-docentes com destaque no campo científico (CONSELHO..., 2013).

Os dados da Figura 1 mostram que os comentários da CA-EF são voltados para incentivar a produção de conhecimento seguindo a lógica do Qualis P. A C1 apareceu com Fr $100 \%$ no RCDo e RCDi. Por sua vez, a cobrança para o corpo docente produzir capital científico foi alta: a C2 obteve uma $\mathrm{Fr}=91,7 \%$ (RCDo). Para o corpo 
discente, os comentários relativos a $\mathrm{C} 2$ apareceram com $\mathrm{Fr}=50 \%$. Os resultados sobre a frequência de comentários relacionados a produção de livros e capítulos foram diferentes. $\mathrm{Na} \mathrm{C} 3$, encontramos uma $\mathrm{Fr}=25 \%$ (RCDo) e Fr=16,7\% (RCDi) (Figura 1).

Mesmo com a implantação da Classificação de Livros a partir da AT-2007, o incentivo para o corpo docente publicar artigos em periódicos com FI/índice h predominou nos comentários da CA-EF presentes nas FA. Como a cobrança para produção de capital científico é maior para o corpo docente, as seguintes expressões foram encontradas no RCDo: "[...] o programa apresenta uma produção internacional compatível com o número de docentes permanentes" (COMISSÃO..., 2007c, p.3): "[...] a publicação de artigos de estratos mais altos é elevada, visto que os estratos A1 e A2 corresponderam a $44 \%$ de toda a produção intelectual contabilizada do programa" (COMISSÃO..., 2010b, p.4); "[...] destaca-se que foram publicados 59 artigos nos estratos superiores (A1 e A2), perfazendo a média de 3,1 artigos A1 ou A2 por docente permanente" (COMISSÃO..., 2010c, p.3); "Sua produção apresenta um número satisfatório em publicações em estratos superiores" (COMISSÃO..., 2010d, p.3). "Destaca-se que há 12 docentes permanentes que publicaram, pelo menos uma produção, nos estratos superiores do Qualis Periódico (A1 e A2)" (COMISSÃO..., 2010e, p.3).

Outras frases apareceram mais de uma vez em diferentes FA. A frase seguinte foi utilizada em três FA: "Há necessidade do Programa concentrar esforços para melhor equilibrar a quantidade de artigos de impacto internacional (IA e IB) entre os docentes permanentes para o triênio seguinte" (COMISSÃO..., 2007e, p.3; 2007f, p.3; 2007g, p.3). Outra frase apareceu duas vezes em FA distintas: "Nota-se que houve um aumento expressivo de produções de artigos em periódicos indexados internacionalmente" (COMISSÃO..., 2010c, p.3; 2010e, p.3). Além disso, a CA-EF destacou no Relatório da AT-2007: "Nota-se que os docentes [...] tem procurado disseminar sua produção em periódicos qualificados no sistema QUALIS" (COMISSÃO..., 2007a, p.12). 
Percebe-se que existe um reconhecimento, por parte da avaliação, do perfil docente com alto capital científico. Desse modo, os bolsistas de Produtividade em Pesquisa (CNPq) obtiveram destaque nos comentários da $\mathrm{CA}-\mathrm{EF}$. A frequência de destaque para a bolsa PQ nos comentários da CA-EF (C4) nas FA foi de 91,7\% (Figura 1), o que representa a importância do alto índice de capital científico individual na composição do capital científico do PPG. No Relatório da AT-2010, a CA-EF deixou claro que:

\footnotetext{
Da mesma forma, e em consonância com agências de fomento, optou-se por valorizar o natural reconhecimento que a comunidade científica atribui aos pesquisadores de reconhecido destaque e liderança científica em suas áreas básicas, de forma a pontuar o corpo docente dos PPGs que contam com docentes que detém bolsa de Produtividade em Pesquisa (Bolsa-PQ) do CNPq (COMISSÃO...., , 2010a, p.3).
}

Segundo o Comitê de Assessoramento de Educação Física, Fonoaudiologia, Fisioterapia e Terapia Ocupacional do CNPq, o processo de seleção de bolsistas PQ adota como critérios gerais de julgamento o volume de artigos publicados em periódicos de bases de dados internacionais e a orientação de mestres e doutores na pós-graduação. Dentre os critérios de classificação e desempate, o mais relevante é o "número de publicações e índices de impacto dos respectivos periódicos científicos nos últimos cinco anos, assim como a posição do pesquisador como autor principal ou orientador" (CONSELHO..., 2013, p.1). De um lado, o pesquisador depende da sua condição de docente na pós-graduação para atender o critério de orientação de mestres e doutores a fim de obter ou manter a bolsa de pesquisador. De outro, a avaliação da CAPES reconhece aqueles PPGs que tem em seu corpo docente pesquisadores-bolsistas PQ, uma vez que seu alto capital científico individual (conquistado com a publicação de artigos em periódicos com FI/índice h) contribuirá com o volume de capital científico do próprio PPG.

Os bolsistas PQs "[... ] são considerados pela política científica brasileira como os mais produtivos academicamente dentro dos 
campos científicos" (CAFÉ; CARVALHO; MENEZES; ODDONE, 2011, p.20). Além de status e reconhecimento no campo científico brasileiro, este tipo de bolsa permite o exercício de poder sobre os mecanismos de acúmulo de capital científico. Indivíduos com alto capital científico conhecem os meios de apropriação simbólica do capital específico e são reconhecidos pelos próprios pares como aqueles que têm condições de ocupar postos que lhes dão o direito de avaliar outros jogadores. Um dos requisitos para que um indivíduo seja membro da Comissão de Área da CAPES, responsável pela avaliação dos PPGs, é "pertencer a programas com, pelo menos, conceito 4, e ter, sempre que possível, bolsa de produtividade de pesquisa do CNPq, prioritariamente nível 1 [o mais elevado]" (COORDENAÇÃO...., 2006, p.6, termo acrescido).

O critério de julgamento do que é (e do que não é) científico passa ser o próprio objeto de disputa no campo científico. Supostamente, o avaliador acaba exercendo, na avaliação, a posição de juiz e jogador. Como juiz, exerce todo seu poder e autoridade sobre os critérios de classificação do capital científico. Como jogador, é parte interessada em conservar a estrutura objetiva de classificação do capital científico que o mantém na posição dominante no campo científico. Os indivíduos que compactuam com as regras do jogo, tem interesse em conservar o que é produzido no campo e com isso, conseguem se conservar conservando funcionamento do próprio campo (BOURDIEU, 1983).

Tem-se, com o Qualis P, um ciclo de reprodução da lógica da produtividade em pesquisa aliado à publicação de artigos em periódicos com FI/índice-h como mecanismo objetivo da produção de capital científico na EF. Por sua vez, a manutenção da lei objetiva de acúmulo de capital científico depende da produção conjunta entre o corpo docente e discente, bem como da preparação de novatos para assimilar a lógica dominante da produção científica da pósgraduação em EF (Tabela 1): 
Tabela 1 - Frequência de comentários sobre a produção conjunta entre corpo docente e orientação de bolsistas de IC (graduação) (AT-2007 e AT-2010)

\begin{tabular}{lllcc}
\hline Recorte & \multicolumn{2}{l}{ Característica } & F* $^{*}$ & $\operatorname{Fr}(\%)^{*}$ \\
\hline \multirow{2}{*}{ RCDoDi } & C5 & & 7 & 58,3 \\
& C6 & & 12 & 100 \\
& & Total & $\mathbf{1 2}$ & $\mathbf{1 0 0}$ \\
\hline
\end{tabular}

Fonte: Adaptado dos Resultados da Avaliação Trienal 2007 e 2010. Fichas de Avaliação do PPGEF/USP, PPGCM/UNESP, PPGEF/UFSC, PPGCMH/UFPR e PPGEF/UGF (COMISSÄO..., 2007b; 2007c; 2007d; 2007e; 2007f; 2007g; 2010b; 2010c; 2010d; 2010e; 2010f; 2010g).

*Frequência absoluta $(\mathrm{F})$ e frequência relativa $(\mathrm{Fr})$

Termos considerados para cada característica:

C5 - Qualis P e a produção conjunta entre docentes e discentes: (Qualquer referência do Qualis P relacionada aos termos) produção conjunta entre docentes e discentes/egressos; produções envolvendo docentes e discentes; produção intelectual (refere-se a produção do PPG e, portanto, do corpo docente) que envolve a participação discente.

C6 - Envolvimento do corpo docente com atividade de alunos de IC: envolvimento do corpo docente com atividade de alunos da graduação com bolsa de Iniciação Científica (IC).

Reconhecendo a cobrança para o corpo docente produzir capital científico (Figura 1), por sua vez existe uma indução a manutenção da produção conjunta entre o corpo docente e discente voltada para a produção de artigos bem ranqueados no Qualis $\mathrm{P}(\mathrm{C} 5-\mathrm{Fr}=58,3 \%)$ (Tabela 1). Com isso, a preparação de novatos na lógica do campo científico da EF também é valorizada na avaliação dos PPGs em EF. A orientação de alunos de IC foi destaque nos comentários dos avaliadores: encontramos uma $\mathrm{Fr}=100 \%$ para a C6 (Tabela 1). A orientação de bolsistas IC tem sido vista como "um passaporte para sua entrada na pós-graduação e um sinal forte de seu interesse na pesquisa" (LOVISOLO, 2003, p.100). 
Quando ex-alunos de IC ingressam nos PPGs como discentes, muitas vezes já incorporaram um sentido prático das regras do jogo científico. Com isso, o sistema de classificação do capital científico pode induzir a adoção do ciclo de trocas de reconhecimento entre indivíduos que possuem capital científico (detentores) e aqueles que estão iniciando a entrada no campo (pretendentes). Os novatos investem no acúmulo de capital adotando estratégias de sucessão. Muitas vezes isso ocorre na construção conjunta do volume de capital entre detentores e pretendentes. O detentor usa todo seu prestígio para alavancar o acúmulo de capital do novato. Em troca, o novato contribui para elevar ainda mais o capital do indivíduo já estabelecido no campo. Esse ciclo reforça as leis objetivas de acúmulo de capital, garantindo a troca, entre detentor e novato, de poder e autoridade sobre os mecanismos do campo. Mantém-se assim a continuidade de uma linhagem e a conservação do próprio campo (BOURDIEU, 1975; 1983).

Mesmo com dois instrumentos de classificação da produção científica (Qualis P e a Classificação de Livros), os dados mostraram que o processo de avaliação da produção científica dos PPGs da EF tem incentivado mais a publicação de artigos bem classificados no Qualis P do que a disseminação no formato de livros/capítulos. A CA-EF reconheceu a importância dos livros/capítulos para grupos que produzem conhecimento vinculado com as ciências humanas e sociais e que a Classificação de Livros foi uma estratégia para amenizar as distorções no processo de classificação da produção científica da área (COMISSÃO..., 2007a; 2009).

O PPGEF/UGF, único programa com redução de nota da AT2007 para a AT-2010 (5 para 4), esclareceu que o formato padronizado do sistema de classificação da produção intelectual (Qualis P) e a adoção de critérios universais ainda não levam em consideração as diferentes características de produção de conhecimento da área, privilegiando o PPG que mais se adéqua a divulgação em periódicos de circulação internacional. Ainda assim, o PPGEF/UGF colocou que o corpo docente investiu na publicação em periódicos do exterior para atender as exigências de publicação. 
O PPGEF/UGF tinha, na época da AT-2007 e AT-1010, a maior parte das linhas de pesquisa voltada para a produção de livros e capítulos (PROGRAMA..., 2007; 2010).

A CA-EF também reconheceu, na AT-2007, a dificuldade que grupos da EF vinculados com as ciências humanas e sociais têm em publicar artigos em periódicos com FI/índice h: "se a hierarquia que se estabelece para os níveis de IA para NB faz sentido para classificar a produção das áreas de concentração de orientação biodinâmica, o mesmo pode não acontecer para as áreas sociocultural e pedagógica" (COMISSÃO..., 2007a, p.14). Na AT-2010, a CA-EF acrescentou outras limitações: (a) a dificuldade em compor uma listagem de periódicos que atenda áreas de produção de pesquisa básica e aplicada; (b) a quantidade de periódicos voltados para publicação de pesquisa aplicada é limitada; e (c) o fator de impacto de periódicos da área social, cultural e pedagógica é menor quando comparado com revistas das áreas biológicas (COMISSÃO ..., 2010a).

Mesmo com a diferença no processo de produção e disseminação do conhecimento existente na área da EF, a alternativa proposta pela CA-EF foi voltada para melhorar a produção de artigos dos grupos vinculados com as ciências humanas e sociais. Como a maior inserção de artigos deste grupo foi em periódicos nacionais, a estratégia sugerida foi incentivar a qualificação dos periódicos nacionais com a adoção do padrão internacional (COMISSÃO...., 2007a). Com isso, dois dos principais periódicos brasileiros da EF estão no JCR. A revista Motriz, listada no JCR Science Edition 2011 foi a única brasileira da $\mathrm{EF}$ a constar na listagem de 84 periódicos classificados da área sport sciences (descritor que mais se aproxima da área). A revista Movimento consta na listagem de outra área de conhecimento, JCR Social Science Edition 2011, classificada como social sciences, interdisciplinar (THOMSON REUTERS, 2012). A Motriz e a Movimento são os periódicos brasileiros da EF mais bem ranqueados no Qualis P: A2 (COORDENAÇÃO..., 2013c).

A adoção do padrão internacional no que diz respeito à produção de artigos estimula os periódicos brasileiros da EF a adotarem as características internacionais, tais como: busca pelo FI/índice h e 
publicação em inglês. Por exemplo, a Motriz passou a publicar artigos somente no idioma inglês (MOTRIZ, 2013). Desse modo, as revistas brasileiras tendem a buscar indexação em bases internacionais para melhorarem sua classificação no Qualis P e pesquisadores tendem a disseminar seus artigos nos periódicos mais bem ranqueados no Qualis P (TANI, 2007).

O Qualis P tem se mostrado como um mecanismo de hierarquização de posições entre os grupos que produzem conhecimento em diferentes vertentes na EF. Também, o Qualis P é composto, na mesma classificação, por periódicos que apresentam dinâmicas de citação diferentes. Periódicos vinculados com as ciências biológicas tendem a ter um maior FI/índice h do que aqueles periódicos com aproximação das ciências humanas e sociais (VITOR-COSTA; MAIA DA SILVA; SORIANO, 2012). Supõese que existe uma pressão para forçar as subáreas da EF a se ajustarem na lógica de produção científica da subárea dominante: o grupo da EF com produção vinculada com as ciências biológicas: biodinâmica.

O que temos na área da EF, com o Qualis P, é o "efeito Mateus" (MERTON, 1968). Os que já possuem capital científico são incentivados a elevarem ainda mais o volume de seu capital. Por outro lado, aqueles que não possuem capital científico, têm as chances de produção deste capital cada vez mais reduzida. $\mathrm{O}$ incentivo a adoção de padrões internacionais por parte dos periódicos brasileiros foi colocada como uma alternativa para amenizar a dificuldade que grupos vinculados com as ciências humanas e sociais têm em publicar em periódicos internacionais. Todavia, esse caminho tem funcionado como um processo de legitimação da regra objetiva de produção de capital científico, favorecendo o produto científico que os dominantes são detentores.

Acreditamos que o Qualis P da EF funciona como estrutura estruturada e estruturante do campo científico da EF. Um sistema estruturado estabelece o princípio lógico que organiza a percepção do campo social (campo científico) em classes/posições hierárquicas. Ao mesmo tempo, é um sistema estruturante que norteia a prática e 
a maneira como essa prática é percebida. Um sistema estruturado e estruturante pode exercer um papel impositivo e de legitimação da dominação de um grupo sobre outro (BOURDIEU, 2004; 2007a; 2007b). Com base nos dados analisados, podemos colocar que o Qualis P da EF funciona como uma estrutura estruturada e, portanto, uma estrutura de exercício de poder sobre a classificação da produção científica dos PPGs da EF. O Qualis P é determinado na lógica objetiva de direcionamento da produção de conhecimento do corpo docente e discente. Ao mesmo tempo, legitima a hierarquização de posições sociais dentro dos PPGs da EF.

\section{Conclusão}

Verificamos, nas Fichas de Avaliação Trienal dos PPGs em EF, a constituição de um ciclo de conservação da regra objetiva de acúmulo de capital científico. O Qualis $\mathrm{P}$, formado a partir da produção científica dos PPGs, representa a tendência dominante de produção científica do corpo docente. Por outro lado, o próprio Qualis $\mathrm{P}$ é empregado para direcionar a produção científica dos PPGs. Garante-se assim que o tipo de produção científica predominante na pós-graduação em EF continue sendo o tipo de produção científica dominante: o da biodinâmica.

Consideramos que a avaliação da produção científica dos PPGs da EF tem um papel fundamental no processo de manutenção das leis objetivas de acúmulo de capital científico, com o Qualis P funcionando como estrutura estruturada. A conservação da estrutura objetiva de produção de capital científico é garantida pelos próprios jogadores (docentes/discentes). Os jogadores, assumindo posição (favorável ou oposta) jogam de acordo com as regras do jogo e compactuam com o princípio lógico do Qualis $\mathrm{P}$ ao investirem na produção de capital científico para garantir sua permanência na pósgraduação.

Os próprios jogadores, ao atuarem como juízes ocupando posições nas comissões de área da CAPES, responsáveis pela avaliação dos PPGs, supostamente, vão procurar reproduzir os 
critérios de julgamento do que eles consideram "científico" (com base no seu capital acumulado), e que, por sua vez, os mantém na posição dominante. Com isso, garantem a conservação da estrutura objetiva de acúmulo de capital científico nos PPGs da EF.

Como restringimo-nos apenas a verificar nos documentos oficiais da Avaliação Trienal dos PPGs em EF (triênios de 2007 e 2010) o funcionamento do Qualis Periódicos na determinação de regras objetivas para obtenção de capital científico, seria necessário investigar, na produção científica disseminada pelo corpo docente e discente, como o Qualis P funciona como estrutura estruturante do processo de acumulação de capital científico, sendo esta a limitação do estudo. 


\begin{abstract}
Qualis Periódicos and the production of scientific capital in Physical Education Graduate Programs

Abstract: Objective: to verify in the official documents of the Triennial Assessment of Physical Education Graduate Programs (triennium of 2007 and 2010) the operation of Qualis Periódicos in the determination of objective rules to acquire scientific capital. Methodology: a qualitative approach and an exploratory study was the most adequate strategy to conduct this research. We selected six graduate programs in Physical Education. We collected data from comments in twelve Triennial Assessment forms of 2007 and 2010. Conclusion: the Qualis P functions as a structured structure establishing the logical principle of accumulation of scientific capital.
\end{abstract}

Keywords: Evaluation. Scientific Production. Graduate Education. Physical Education.
Qualis Periódicos y la producción de capital científico en los Programas de Posgrado en Educación Física

Resumen: Objetivo: verificar en los documentos oficiales de la Evaluación Trienal de los Posgrados en Educación Física (período de 2007 y 2010) el funcionamiento de Qualis Periódicos para determinar las reglas objetivas para la obtención de capital científico. Metodología: el foco cualitativo y el estudio exploratorio fue la mejor forma hacia conducir el estudio. Seleccionamos seis programas de postgrado en Educación Física. Colectamos el datos en comentários de doce fichas de la Evaluación del años 2007 y 2010. Conclusión: Qualis P funciona como estructura estructurada que se establece la lógica de la acumulación de capital científico.

Palabras-clave: Evaluación. Producción Científica. Posgrado. Educación Física. 


\section{REFERÊNCIAS}

BARDIN, Laurence. Análise de conteúdo. Lisboa: Edições 70, 1977.

BOURDIEU, Pierre. A distinção: crítica social do julgamento. São Paulo: Edusp; Porto Alegre: Zouk, 2007a.

BOURDIEU, Pierre. Homos academicus. Florianópolis: UFSC, 2011.

BOURDIEU, Pierre. O campo científico. In: ORTIZ, R. (Org.). Bourdieu. São Paulo: Ática, 1983. p.82-121. (Coleção Grandes Cientistas Sociais, n.39)

BOURDIEU, Pierre. O poder simbólico. 11. ed. Rio de Janeiro: Bertrand Brasil, 2007b.

BOURDIEU, Pierre. Os usos sociais da ciência. São Paulo: UNESP, 2004.

BOURDIEU, Pierre. The specificity of the scientific field and the social conditions of the progress of reason. Social Science Information, Paris, v.14, n.6, p.19-47, jan. 1975.

BRASIL. Plano Nacional de Pós-Graduação (PNPG 2011-2020). Coordenação de Aperfeiçoamento de Pessoal de Nível Superior (CAPES). Brasília/DF-CAPES, 2010.

BUTLER, Declan. Free journal-ranking tool enters citation market. Nature, London, v.451, n.7174, p.6, 2008.

CAFÉ, Anderson; CARVALHO, Kátia de; MENEZES, Vinícios; ODDONE, Nanci. A elite acadêmica da sociologia no Brasil e sua produção científica. Informação \& Informação, Londrina, v.16, n.3, p.19-39, jan./jun. 2011.

CENTRO DE GESTÃO E ESTUDOS ESTRATÉGICOS. Doutores 2010: estudos da demografia da base técnico-científica brasileira. Centro de Gestão e Estudos Estratégicos (CGEE). Brasília: CGEE. 2010.

COMISSÃO DE AVALIAÇÃO - EDUCAÇÃO FíSICA. Documento de área 2007. Área de avaliação: Educação Física, Fisioterapia, Fonoaudiologia e Terapia Ocupacional. Comissão de Avaliação - Educação Física (CA-EF). Coordenação de Aperfeiçoamento de Pessoal de Nível Superior. Brasília, 2007a. Disponível em: <http://www.capes.gov.br/images/stories/download/avaliacaotrienal/ doc_areas_trienal_2007/2007_EducacaoFisica_Aval2004-2006.pdf >. Acesso em: 11 jun. 2013.

COMISSÃO DE AVALIAÇÃO - EDUCAÇÃO FíSICA. Documento de área 2009. Área de avaliação: Educação Física, Fisioterapia, Fonoaudiologia e Terapia Ocupacional. Comissão de Avaliação - Educação Física (CA-EF). Coordenação de Aperfeiçoamento de Pessoal de Nível Superior. Brasília, 2009. Disponível em: <http:/ /www.capes.gov.br/images/stories/download/avaliacao/EDU_FIS15out2009.pdf>. Acesso em: 11 jun. 2013. 
COMISSÃO DE AVALIAÇÃO - EDUCAÇÃO FÍSICA. Relatório de avaliação 20072009 trienal 2010. Comissão de Avaliação - Educação Física (CA-EF). Coordenação de Aperfeiçoamento de Pessoal de Nível Superior (CAPES). Brasília, 2010a. Disponível em: <http://trienal.capes.gov.br/wp-content/uploads/2011/03/RelatorioFinal-Area-21.pdf>. Acesso em: 11 jun. 2013.

COMISSÃO DE AVALIAÇÃO - EDUCAÇÃO FÍSICA. Resultados da Avaliação Trienal 2007. Ficha de Avaliação do Programa de Pós-Graduação em Educação Física da Universidade de São Paulo. Comissão de Avaliação - Educação Física. Coordenação de Aperfeiçoamento de Pessoal de Nível Superior (CAPES). Brasília, 2007b. Disponível em: <http://conteudoweb.capes.gov.br/conteudoweb/ VisualizadorServlet?nome $=33002010 / 021 / 2006$ _021_33002010084P9_Ficha.pdf \&aplicacao $=$ avaliacaotrienal\&idEtapa $=2 \& a n o=2006 \&$ tipo $=$ divulga $>$. Acesso em: 20 fev. 2010.

COMISSÃO DE AVALIAÇÃO - EDUCAÇÃO FÍSICA. Resultados da Avaliação Trienal 2007. Ficha de Avaliação do Programa de Pós-Graduação em Ciências da Motricidade da Universidade Estadual Paulista Júlio de Mesquita Filho. Comissão de Avaliação - Educação Física. Coordenação de Aperfeiçoamento de Pessoal de Nível Superior (CAPES). Brasília, 2007c. Disponível em: <http:// conteudoweb.capes.gov.br/conteudoweb/VisualizadorServlet?nome=33004137/ 021/2006_021_33004137062P0_Ficha.pdf\&aplicacao=avaliacaotrienal\& idEtapa $=2 \&$ ano=2006\&tipo=divulga $>$. Acesso em: 20 fev. 2010.

COMISSÃO DE AVALIAÇÃO - EDUCAÇÃO FÍSICA. Resultados da Avaliação Trienal 2007. Ficha de Avaliação do Programa de Pós-Graduação em Educação Física da Universidade Federal de Santa Catarina. Comissão de Avaliação Educação Física. Coordenação de Aperfeiçoamento de Pessoal de Nível Superior (CAPES). Brasília, 2007d. Disponível em: <http://conteudoweb.capes.gov.br/ conteudoweb/VisualizadorServlet? nome $=41001010 / 021 /$ 2006_021_41001010039P3_Ficha.pdf\&aplicacao $=$ avaliacaotrienal\&idEtapa $=$ 2\&ano=2006\&tipo=divulga $>$. Acesso em: 20 fev. 2010.

COMISSÃO DE AVALIAÇÃO - EDUCAÇÃO FÍSICA. Resultados da Avaliação Trienal 2007. Ficha de Avaliação do Programa de Pós-Graduação em Ciências do Movimento Humano da Universidade Federal do Rio Grande do Sul. Comissão de Avaliação - Educação Física. Coordenação de Aperfeiçoamento de Pessoal de Nível Superior (CAPES). Brasília, 2007e. Disponível em: <http:// conteudoweb.capes.gov.br/conteudoweb/VisualizadorServlet?nome=42001013/ 021/2006_021_42001013051P2_Ficha.pdf\&aplicacao=avaliacaotrienal\&idEtapa= $2 \&$ ano $=2006 \&$ tipo $=$ divulga $>$. Acesso em: 20 fev. 2010 .

COMISSÃO DE AVALIAÇÃO - EDUCAÇÃO FÍSICA. Resultados da Avaliação Trienal 2007. Ficha de Avaliação do Programa de Pós-Graduação em Educação Física - Universidade Federal do Paraná. Comissão de Avaliação - Educação Física. Coordenação de Aperfeiçoamento de Pessoal de Nível Superior (CAPES). Brasília, 2007f. Disponível em: <http://conteudoweb.capes.gov.br/conteudoweb/ $\mathrm{V}$ i s u a I i z a d or ServIet? nom e = 40001016/021/ 2006_021_40001016047P0_Ficha.pdf\&aplicacao=avaliacaotrienal\&idEtapa= $2 \& a n o=2006 \&$ tipo=divulga $>$. Acesso em: 20 fev. 2010 .

Movimento, Porto Alegre, v. 20, n. 01, p. 281-304, jan/mar de 2014. 
COMISSÃO DE AVALIAÇÃO - EDUCAÇÃO FíSICA. Resultados da Avaliação Trienal 2007. Ficha de Avaliação do Programa de Pós-Graduação em Educação Física da Universidade Gama Filho. Comissão de Avaliação - Educação Física. Coordenação de Aperfeiçoamento de Pessoal de Nível Superior (CAPES). Brasília, 2007g. Disponível em: <http://conteudoweb.capes.gov.br/conteudoweb/ VisualizadorServlet $?$ nome $=31006019 / 021 / 2006$ 021_31006019004P5 Ficha. df \&aplicacao $=$ avaliacaotrienal\&idEtapa $=2 \& a n o=2006 \&$ tipo $=$ divulga $>$. Acesso em: 20 fev. 2010.

COMISSÃO DE AVALIAÇÃO - EDUCAÇÃO FÍSICA. Resultados da Avaliação Trienal 2010. Ficha de Avaliação do Programa de Pós-Graduação em Educação Física da Universidade de São Paulo. Comissão de Avaliação - Educação Física. Coordenação de Aperfeiçoamento de Pessoal de Nível Superior (CAPES). Brasília, 2010b. Disponível em: <http://trienal.capes.gov.br/wp-content/uploads/2010/12/ fichas/33002010084P9.pdf>. Acesso em: 20 fev. 2013.

COMISSÃO DE AVALIAÇÃO - EDUCAÇÃO FÍSICA. Resultados da Avaliação Trienal 2010. Ficha de Avaliação do Programa de Pós-Graduação em Ciências da Motricidade da Universidade Estadual Paulista Júlio de Mesquita Filho. Comissão de Avaliação - Educação Física. Coordenação de Aperfeiçoamento de Pessoal de Nível Superior (CAPES). Brasília, 2010c. Disponível em: <http://trienal.capes.gov.br/ wp-content/uploads/2010/12/fichas/33004137062P0.pdf>. Acesso em: 20 fev. 2013.

COMISSÃO DE AVALIAÇÃO - EDUCAÇÃO FÍSICA. Resultados da Avaliação Trienal 2010. Ficha de Avaliação do Programa de Pós-Graduação em Educação Física da Universidade Federal de Santa Catarina. Comissão de Avaliação - Educação Física. Coordenação de Aperfeiçoamento de Pessoal de Nível Superior (CAPES). Brasília, 2010d. Disponível em: <http://trienal.capes.gov.br/wp-content/uploads/2010/12/ fichas/41001010039P3.pdf>. Acesso em: 20 fev. 2013.

COMISSÃO DE AVALIAÇÃO - EDUCAÇÃO FíSICA. Resultados da Avaliação Trienal 2010. Ficha de Avaliação do Programa de Pós-Graduação em Ciências do Movimento Humano da Universidade Federal do Rio Grande do Sul. Comissão de Avaliação - Educação Física. Coordenação de Aperfeiçoamento de Pessoal de Nível Superior (CAPES). Brasília, 2010e. Disponível em: <http://trienal.capes.gov.br/ wp-content/uploads/2010/12/fichas/42001013051P2.pdf>. Acesso em: 20 fev. 2013.

COMISSÃO DE AVALIAÇÃO - EDUCAÇÃO FÍSICA. Resultados da Avaliação Trienal 2010. Ficha de Avaliação do Programa de Pós-Graduação em Educação Física - Universidade Federal do Paraná. Comissão de Avaliação - Educação Física. Coordenação de Aperfeiçoamento de Pessoal de Nível Superior (CAPES). Brasília, 2010f. Disponível em: <http://trienal.capes.gov.br/wp-content/uploads/2010/12/ fichas/40001016047P0.pdf>. Acesso em: 20 fev. 2013.

COMISSÃO DE AVALIAÇÃO - EDUCAÇÃO FÍSICA. Resultados da Avaliação Trienal 2010. Ficha de Avaliação do Programa de Pós-Graduação em Educação Física da Universidade Gama Filho. Comissão de Avaliação - Educação Física. Coordenação de Aperfeiçoamento de Pessoal de Nível Superior (CAPES). Brasília, 2010g. Disponível em: <http://trienal.capes.gov.br/wp-content/uploads/2010/12/ fichas/31006019004P5.pdf>. Acesso em: 20 fev. 2013. 
CONSELHO NACIONAL DE DESENVOLVIMENTO CIENTÍFICO E TECNOLÓGICO. Critérios de julgamento do comitê de assessoramento área 21- bolsa produtividade em pesquisa. Conselho Nacional de Desenvolvimento Científico e Tecnológico -CNPq. Brasília, 2013. Disponível em: <http://cnpq.br/web/guest/ view/-/journal_content/56_INSTANCE_0oED/10157/49725>. Acesso em: 30 mai. 2013.

COORDENAÇÃO DE APERFEIÇOAMENTO DE PESSOAL DE NÍVEL SUPERIOR. Caderno de Indicadores. Coordenação de Aperfeiçoamento de Pessoal de Nível Superior (CAPES). Brasília, 2013a. Disponível em: <http:// conteudoweb.capes.gov.br/conteudoweb/CadernoAvaliacaoServlet>. Acesso em: 21 out. 2013.

COORDENAÇÃO DEAPERFEIÇOAMENTO DE PESSOALDENIVELSUPERIOR. Qualis Periódicos. Coordenação de Aperfeiçoamento de Pessoal de Nível Superior (CAPES). Brasília, 2013b. Disponível em: <http://www.capes.gov.br/avaliacao/ qualis>. Acesso em: 23 mai. 2013.

COORDENAÇÃO DE APERFEIÇOAMENTO DE PESSOAL DE NÍVEL SUPERIOR. Regulamento para avaliação trienal 2010 (2007-2009). Coordenação de Aperfeiçoamento de Pessoal de Nível Superior (CAPES). Brasília, 2006. Disponível em: <http://trienal.capes.gov.br/wp-content/uploads/2010/07/REGULAMENTOPARA-A-AVALIA\%C3\%87\%C3\%830-09jul10.pdf>. Acesso em: 23 mai. 2013.

COORDENAÇÃO DE APERFEIÇOAMENTO DE PESSOAL DE NÍVEL SUPERIOR. Webqualis 2012. Área de avaliação: Educação Física. Coordenação de Aperfeiçoamento de Pessoal de Nível Superior (CAPES). Brasília, 2013c. Disponível em: <http://qualis.capes.gov.br/webqualis/publico/documentosDeArea.seam>. Acesso em: 29 abr. 2013.

COSTA, Dirceu. Apresentação. In: FÓRUM NACIONAL DE PÓS-GRADUAÇÃO EM EDUCAÇÃO FÍSICA, FONOAUDIOLOGIA, FISIOTERAPIAE TERAPIA OCUPACIONAL. São Paulo: jun. 2009. Disponível em: <http://fefnet172.fef.unicamp.br/posgraduacao/ forumpg/arquivos/forum/documentos/Reuniao_Sao_Paulo_2009/FORUM_46_na_UNIFESP-SP.ppt>. Acesso em: 01 ago. 2009.

CRESWELL, John W. Projeto de pesquisa: métodos qualitativo, quantitativo e misto. 2.ed. Porto Alegre: Artmed e Bookman, 2007.

DAVYT, Ailcar; VELHO, Léa. A avaliação da ciência e a revisão por pares: passado e presente. Como será o futuro? História, Ciências, Saúde, Rio de Janeiro, v.7, n.1, p.93-116, 2000.

DENZIN, Norman K.; LINCON, Yvonna S. O planejamento da pesquisa qualitativa. Teorias e abordagens. 2.ed. Porto Alegre: Artmed, 2006.

LOVISOLO, Hugo Rodolfo. A política de pesquisa e a mediocridade possível. Revista Brasileira de Ciências do Esporte, Campinas, v.24, n.2, p.97-114, 2003.

MARCHLEWSKI, Camila; MAIA DA SILVA, Priscilla; SORIANO, Jeane Barcelos. A influência do sistema de avaliação Qualis na produção de conhecimento científico: 
algumas reflexões sobre a educação física. Motriz, Rio Claro, v.17, n.1, p. 104-11 , 2011.

MERTON, Robert.K. The Matthew effect in science. Science, Washington-DC, v.159, n.3810, p.56-63, 1968.

MINAYO, Maria Cecília de Souza. O desafio do conhecimento. Pesquisa qualitativa em saúde. 5.ed. São Paulo: Hucitec-Abrasco, 1998.

MOTRIZ. Author Guidelines. Rio Claro, 2013. Disponível em: <http:// www.periodicos.rc.biblioteca.unesp.br/index.php/motriz/about/ submissions\#authorGuidelines>. Acesso em: 10 jun. 2013.

PROGRAMA DE PÓS-GRADUAÇÃO EM EDUCAÇÃO FÍSICA DA UNIVERSIDADE GAMA FILHO. Proposta do Programa - 2007. Caderno de Indicadores. Programa de Pós-Graduação em Educação Física da Universidade Gama Filho. Coordenação de Aperfeiçoamento de Pessoal de Nível Superior (CAPES). Brasília, 2007. Disponível em: <http://conteudoweb.capes.gov.br/conteudoweb/VisualizadorServlet?nome= 2007/31006019/021/2007_021_31006019004P5_Proposta.pdf\&aplicacao= cadernoavaliacao>. Acesso em: 23 mai. 2013.

PROGRAMA DE PÓS-GRADUAÇÃO EM EDUCAÇÃO FÍSICA DA UNIVERSIDADE GAMA FILHO. Proposta do Programa - 2010. Caderno de Indicadores. Coordenação de Aperfeiçoamento de Pessoal de Nível Superior (CAPES). Brasília, 2010. Disponível em: <http://conteudoweb.capes.gov.br/conteudoweb/ Visualizadorservlet? nom e =2009/31006019/021/ 2009_021_31006019004P5_Proposta.pdf\&aplicacao=cadernoavaliacao >. Acesso em: 23 mai. 2013.

RICHARDSON, Roberto Jarry. Pesquisa social: Métodos e técnicas. 3.ed. São Paulo: Atlas, 2008.

SOUZA, Elaine Pereira de; PAULA, Maria Carlota de Souza. Qualis: a base de qualificação dos periódicos científicos utilizada na avaliação Capes. Infocapes, Boletim Informativo, Brasília, v.10, n.2, p.7-25 , abr./jun. 2002.

TANI, Go. Educação física: por uma política de publicação visando a qualidade dos periódicos. Revista Brasileira de Ciências do Esporte, Campinas, v.29, n.1, p.9-22, 2007.

THOMSON REUTERS. JCR Science and Social Science Edition 2011. Disponível em: <http://admin-apps.webofknowledge.com.ez78.periodicos.capes.gov.br/JCR/ JCR?PointOfEntry=Home\&SID=2CZuayjdS7It2WSsg1K>. Acesso em: 04 ago. 2012.

VITOR-COSTA, Marcelo; MAIA DA SILVA, Priscilla; SORIANO, Jeane Barcelos. A avaliação da produtividade em pesquisa na Educação Física: reflexões sobre algumas limitações dos indicadores bibliométricos. Revista Brasileira de Educação Física e Esporte, São Paulo, v.26, n.4, p.581-597, 2012. 


\section{ArtigosOriginais}

Endereço para correspondência

Priscilla Maia da Silva

Rua Benjamim Constant, 1369, apto 4, Centro.

CEP: 86020-320.

Londrina-Paraná. Brasil

Recebido em: 05.07.2013

Aprovado em: 02.10.2013

Movimento, Porto Alegre, v. 20, n. 01, p. 261-280,jan/mar de 2014. 